\title{
Les anticorps monoclonaux anti-tumoraux
}

$>$ Les anticorps monoclonaux (AcM) ciblant les tumeurs sont aujourd'hui largement utilisés pour le traitement de patients atteints de cancer et leur nombre est en constante augmentation. Au cours de ces dix dernières années, de nombreuses études ont montré que l'action anti-tumorale de ces anticorps dépasse largement celle de simples thérapies passives comme cela avait été décrit initialement, avec non seulement le recrutement de cellules immunitaires innées pour favoriser l'activation des étapes précoces de la réponse immunitaire mais aussi avec la génération d'une réponse mémoire anti-tumorale protectrice sur le long-terme. La compréhension de ces mécanismes a récemment conduit au développement clinique d'une nouvelle génération d'AcM anti-tumoraux, modifiés afin d'augmenter leurs capacités à interagir avec les cellules immunitaires. Enfin, les premières études précliniques et cliniques ont démontré l'intérêt de développer des combinaisons thérapeutiques associant ces AcM anti-tumoraux à des immuno-, chimio- ou radiothérapies, afin de renforcer leur potentiel immunomodulateur et d'assurer une protection anti-tumorale efficace et durable. <

Du ciblage de la cellule tumorale au recrutement des cellules immunitaires

Dès le début du siècle dernier, l'immunologiste Paul Ehrlich considérait les anticorps comme des molécules à fort potentiel thérapeutique en les baptisant «balles magiques ». Cette vision a pris tout son sens en 1975, avec la découverte de la technique d'obtention d'hybridomes par Georges Köhler et César Milstein et la production d'anticorps monoclonaux (AcM). Peu après cette découverte, le succès de la première utilisation d'un $A c M$ en clinique dans le traitement du lymphome $B[1]^{1}$,

${ }^{1}$ Le premier pour la transplantation (Orthoclone-0KT3), le second pour l'hémostase (Reopro).

\section{Nouvelles perspectives pour générer une réponse immunitaire protectrice et durable}

Claire Deligne ${ }^{1}$, Laurent Gros ${ }^{2}$

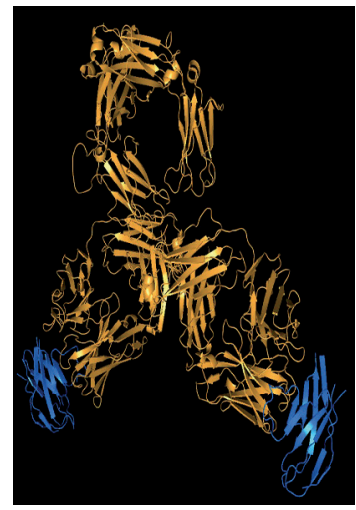

${ }^{1}$ Kennedy Institute

of Rheumatology,

Nuffield Department of

Orthopaedics, Rheumatology and Musculoskeletal Sciences, University of Oxford, Oxford, Royaume-Uni.

${ }^{2}$ IRCM, Institut de Recherche en Cancérologie de Montpellier, Inserm U1194, Université Montpellier, Institut Régional du Cancer de Montpellier, F-34298 Montpellier, France. laurent.gros@inserm.fr

confirmait ces prédictions et ouvrait la voie à l'utilisation thérapeutique d'autres AcM. Les essais cliniques se sont rapidement enchaînés et les premiers succès notables ont vu le jour dans les années 1990 grâce à l'essor de l'ingénierie génétique permettant de transformer progressivement les anticorps murins en anticorps chimériques, puis en anticorps humanisés et, enfin, en anticorps entièrement humains, entraînant une diminution de leur immunogénicité et une augmentation de leur efficacité. Dès lors, leur intérêt thérapeutique va s'accroître tout d'abord dans le cadre de pathologies auto-immunes et inflammatoires, infectieuses, cardiovasculaires, puis dans le traitement de tumeurs malignes hématologiques et solides (Figure IA). En effet, du fait de leur sélectivité pour leurs cibles et de leur affinité élevée, les AcM deviennent un outil thérapeutique de choix pour éliminer de manière spécifique les cellules tumorales tout en épargnant les tissus sains environnants. À la fin de l'année 2017, on enregistre ainsi 73 anticorps ayant obtenu une autorisation de mise sur le marché, dont 34 sont à usage anticancéreux (Figure IA). Depuis l'autorisation de mise sur le marché américain par la FDA (food and drug administration) du rituximab en 1997, utilisé dans les traitements de lymphomes non-Hodgkiniens (LNH), de nombreux autres AcM ciblant la cellule tumorale sont apparus en clinique, dont 25 depuis 2009 (Figure 1B) [2]. Parmi ces anticorps, une majorité ont pour cible la cellule tumorale et sont spécifiques d'un antigène de surface surexprimé par les cellules malignes. Les trois cibles représentées majoritairement sont la molécule CD20, avec quatre anticorps utilisés dans le LNH et les leucémies lymphocytes chroniques B (LLC), le récepteur de l'EGF (epidermal growth factor receptor, EGFR) avec trois anticorps utilisés 


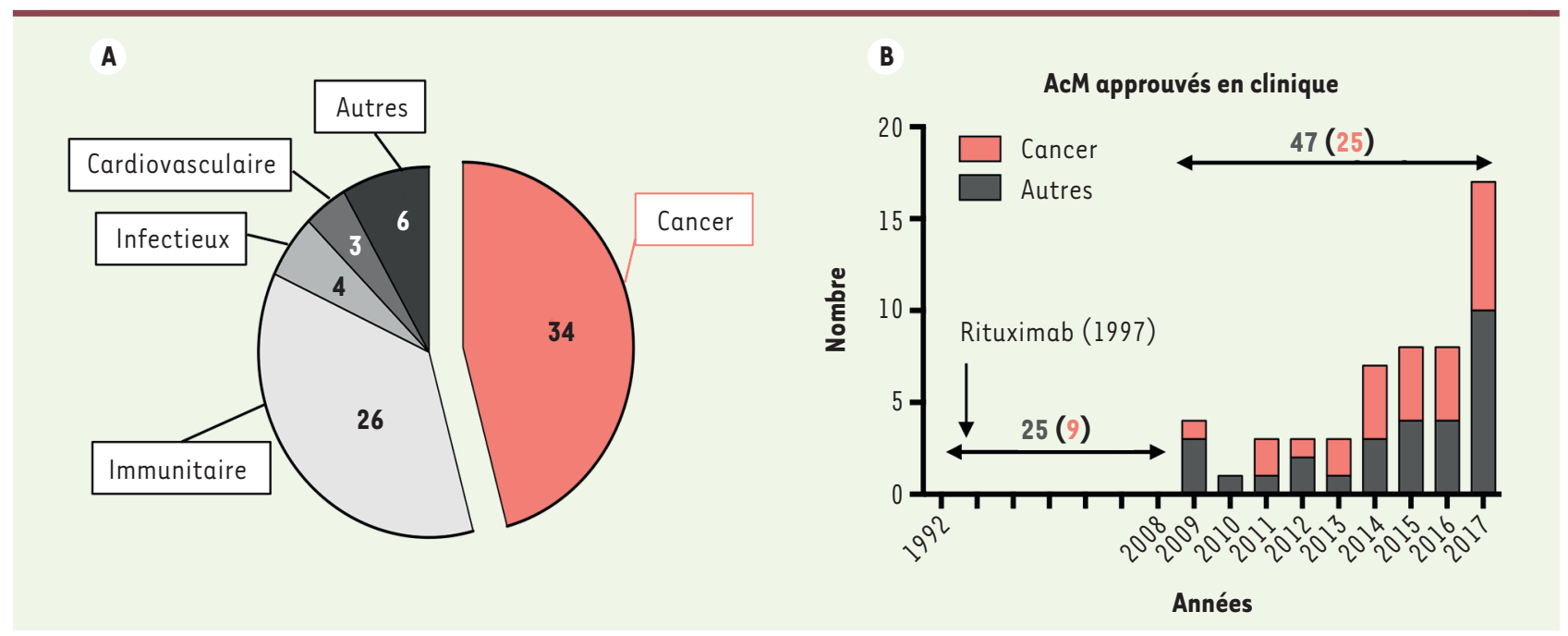

Figure 1. Évolution clinique et impact des AcM anti-tumoraux. Répartition des AcM homologués en clinique en 2017. B. Évolution sur les neuf dernières années du nombre d'AcM homologués en clinique pour les applications en oncologie.

dans les cancer colorectaux (CC) et les cancers du poumon non à petites cellules (CPNPC), et le récepteur HER2/neu de la famille des $\varepsilon G F R$, avec trois anticorps utilisés dans les cancers du sein. De nouveaux AcM dirigés contre de nouvelles cibles sont actuellement en essais cliniques et s'ajouteront certainement dans les années à venir à la liste des AcM thérapeutiques anti-tumoraux.

Un autre grand tournant dans l'histoire des AcM ciblant les tumeurs a été l'émergence de l'idée que l'utilisation d'AcM anti-tumoraux conduisaient à une mobilisation de cellules du système immunitaire des patients, en particulier par l'intermédiaire de l'engagement des récepteurs pour la région Fc des IgG (RFc $\gamma$ ) [3], et aux premières données montrant la mise en place d'une réponse adaptative anti-tumorale [4]. Durant de nombreuses années, chercheurs et cliniciens s'étaient en effet principalement focalisés sur l'étude des effets directs des AcM sur la cellule tumorale (Figure 2), dépendant de la partie Fab de l'anticorps, responsable de la fixation à l'antigène. Ces effets incluent l'inhibition de l'activité de récepteurs membranaires, la neutralisation d'activités enzymatiques, conduisant à la modulation de voies de signalisation et induisant l'activation de voies apoptotiques. L'utilisation d'AcM comme véhicules permettant d'apporter des toxines, des drogues ou des radioéléments à proximité de la cellule cible afin de provoquer sa destruction est un autre exemple des effets directs anti-tumoraux. Les effets indirects, dépendant de la région $\mathrm{Fc}_{c}$ de ces $\mathrm{AcM}_{\mathrm{M}}$ (qui sont en très grande majorité des $\lg G$ ), consistent quant à eux à recruter des cellules ou des molécules du système immunitaire du patient provoquant la mort de la cellule cible par différents mécanismes : ADCC (antibody-dependent cell cytotoxicity), CDC (complement-dependent cytotoxicity), et ADCP (antibody-dependent cell phagocytosis) (Figure 2). Enfin, la caractérisation des effets immunomodulateurs indirects dépendants de la région Fc des $A c M$, démontrant leur capacité à induire une réponse immunitaire anti-tumorale efficace et durable, constitue la véritable avancée des dix dernières années pour les stratégies thérapeutiques anti-cancéreuse (Figure 2).

\section{Des molécules à fort potentiel immunomodulateur}

Un grand nombre d'AcM à usage thérapeutique sont des IgG comportant la région $\mathrm{Fc}$ des $\operatorname{Ig} \mathrm{G} I$ humaines capables d'interagir avec les récepteurs de la région $\mathrm{Fc}$ des IgG ou RFc $\gamma$. Cette interaction est essentielle à l'initiation d'une réponse immunitaire par les cellules de l'immunité innée qui expriment ces RFc $\gamma$ (macrophages, cellules dendritiques [DC], cellules NK [natural killer]). L'impact de ces RFc $\gamma$ et de leur polymorphisme sur le pronostic des patients cancéreux suggère l'implication de mécanismes immunitaires dans les effets cliniques observés. II a par exemple été montré que des patients traités par des AcM anti-CD2O (rituximab) ou anti-HER2/neu (trastuzumab) ont une réponse thérapeutique plus importance lorsqu'ils possèdent une valine en position $158 \mathrm{du}$ RFcyllla (CD16a) et non une phénylalanine $[5,6]$, une substitution qui confère à ce récepteur essentiellement exprimé par les cellules NK et les cellules myéloïdes (monocytes, DC et macrophages), une affinité accrue pour les IgG. Cette observation souligne non seulement l'importance du RFcyllla dans la réponse clinique aux AcM anti-tumoraux, mais également l'importance de I'ADCC qui, par l'intermédiaire des cellules NK, conduit non seulement à la lyse de la cellule cible mais également à la sécrétion d'IFN- $\gamma$ (interféron- $\gamma$ ), une des cytokines clés de la réponse immunitaire anti-tumorale. Cette découverte a d'ailleurs été mise en exergue par le développement d'une nouvelle génération d'anticorps d'isotype IgGl dont les fonctions effectrices ont été modifiées en agissant sur leur glycosylation. Les IgGl humaines possèdent en effet une chaîne de carbohy- 


\section{Effets anti-tumoraux directs}

- Agoniste d'un récepteur, mort cellulaire - Neutralisation d'une activité enzymatique,

- Antagoniste d'un récepteur, blocage

de la dimérisation, réduction de

la prolifération, induction de la mort

cellulaire

\section{Effets anti-tumoraux indirects}

- Cytotoxicité cellulaire dépendante

de l'anticorps: ADCC

- Cytotoxicité dépendante du complément : CDC

- Phagocytose cellulaire dépendante

de l'anticorps: ADCP

abrogation de la signalisation

- Conjugué cytotoxique (drogues, toxines

radio-isotopes) entraînant la mort

cellulaire

Figure 2. Mode d'action des AcM anti-tumoraux. Les AcM agissent par trois mécanismes distincts : (1) les effets anti-tumoraux directs dépendant du Fab, liés à la fixation de l'AcM sur sa cible ; (2) les effets anti-tumoraux indirects qui reposent sur la région Fc, impliquant le recrutement de cellules du système immunitaire exprimant des RFc $\gamma$; et (3) les effets immunomodulateurs dépendant de la région Fc à l'origine des effets vaccinaux.

drates au niveau de l'asparagine 297 de leur chaîne lourde, dont la composition impacte leurs fonctions effectrices [7] ainsi que l'activation des lymphocytes $B$ [8]. L'ingénierie de la région Fc des AcM est ainsi devenue un enjeu majeur et vise à moduler l'affinité des AcM pour les RFc $\gamma$ et à augmenter leurs fonctions effectrices [9, 10]. Ce concept de glyco-ingénierie des AcM s'est traduit par l'introduction en clinique de deux anticorps anti-CD20 dont l'activité cytotoxique est augmentée, l'un afucosylé (l'obinutuzumab), l'autre très faiblement fucosylé (l'ublituximab), utilisés dans le traitement de la LLC [11-13]. De nombreuses autres études de glyco-ingénierie sont en cours sur d'autres anticorps utilisés en clinique, comme les AcM anti-HعR2/neu [14]. Par ailleurs, le système du complément joue également un rôle important dans la mise en place d'une réponse immunitaire dépendante de la région Fc de certains $A_{c} M$. En effet, c'est sur cette région Fc que se fixe le premier composant de la voie classique du complément, le Clq, lorsque l'anticorps est lui-même fixé à son antigène cible, conduisant non seulement à la lyse de la cellule exprimant cet antigène suite à la formation du complexe d'attaque membranaire, mais aussi à la mobilisation et l'activation des cellules immunitaires par le complément (Figure 3). L'importance de ce mécanisme a été exploité avec le développement d'un anticorps anti-CD20, l'ofatumumab, optimisé pour ses capacités à induire une cytotoxicité dépendante du complément (CDC) et utilisé en clinique depuis 2009 pour le traitement de la LLC [15]. Outre leur rôle dans les mécanismes d'ADCC et de CDC, les RFcyl (CD64) et RFcyllI (CD16) confèrent aux cellules présentatrices d'antigène (CPA) qui les expriment la capacité de phagocyter les cellules cibles opsonisées ${ }^{2}$ ainsi que les complexes immuns $(\mathrm{Cl})$ formés de fragments de cellules tumorales complexées avec l'AcM thérapeutique, après une lyse cellulaire de type ADCC ou CDC. Le rôle des $\mathrm{Cl}$ cellule-cible/AcM dans

\footnotetext{
${ }^{2}$ Recouvertes d'anticorps qui les ciblent et se fixent aux récepteurs.
}

la mise en place de réponses immunitaires vaccinales a été montré dans un modèle de leucémie viro-induite [16], dans lequel la captation de ces complexes immuns entraîne une meilleure activation des CPA et une présentation antigénique optimisée (Figure 3). L'importance de ce mécanisme a notamment été montrée lors du traitement de patients présentant un myélome multiple par un anticorps, le daratumumab, dirigé contre CD38 [17], une molécule surexprimée par les cellules de myélome et des plasmocytes normaux lors de la maturation de ces derniers à partir de plasmablastes. De même, l'activation des cellules NK par un traitement avec un anticorps antiEGFR conduit à la maturation des cellules dendritiques et à l'activation de lymphocytes T spécifiques de la tumeur [18]. Cette interaction croisée entre cellules NK et cellules dendritiques favorise la maturation de ces dernières et potentialise la production d'IL(interleukine)-12 nécessaire à l'établissement d'une immunité anti-tumorale à long terme [19]. De récents travaux menés dans un modèle murin de mélanome montre qu'une immunothérapie utilisant un AcM ciblant la protéine TYRPI (tyrosinase-related protein 1 ), une enzyme responsable de la production de mélanine, entraîne, peu de temps après le traitement, une immunomodulation qui se traduit par une augmentation de la production d'IL-12 par les macrophages et les cellules dendritiques, et une augmentation de la production d'IFN- $\gamma$ par les cellules NK [20]. Cette immunomodulation précoce est à l'origine de l'établissement d'une réponse adaptative humorale et cellulaire mémoire capable de protéger les souris sur le long-terme. 


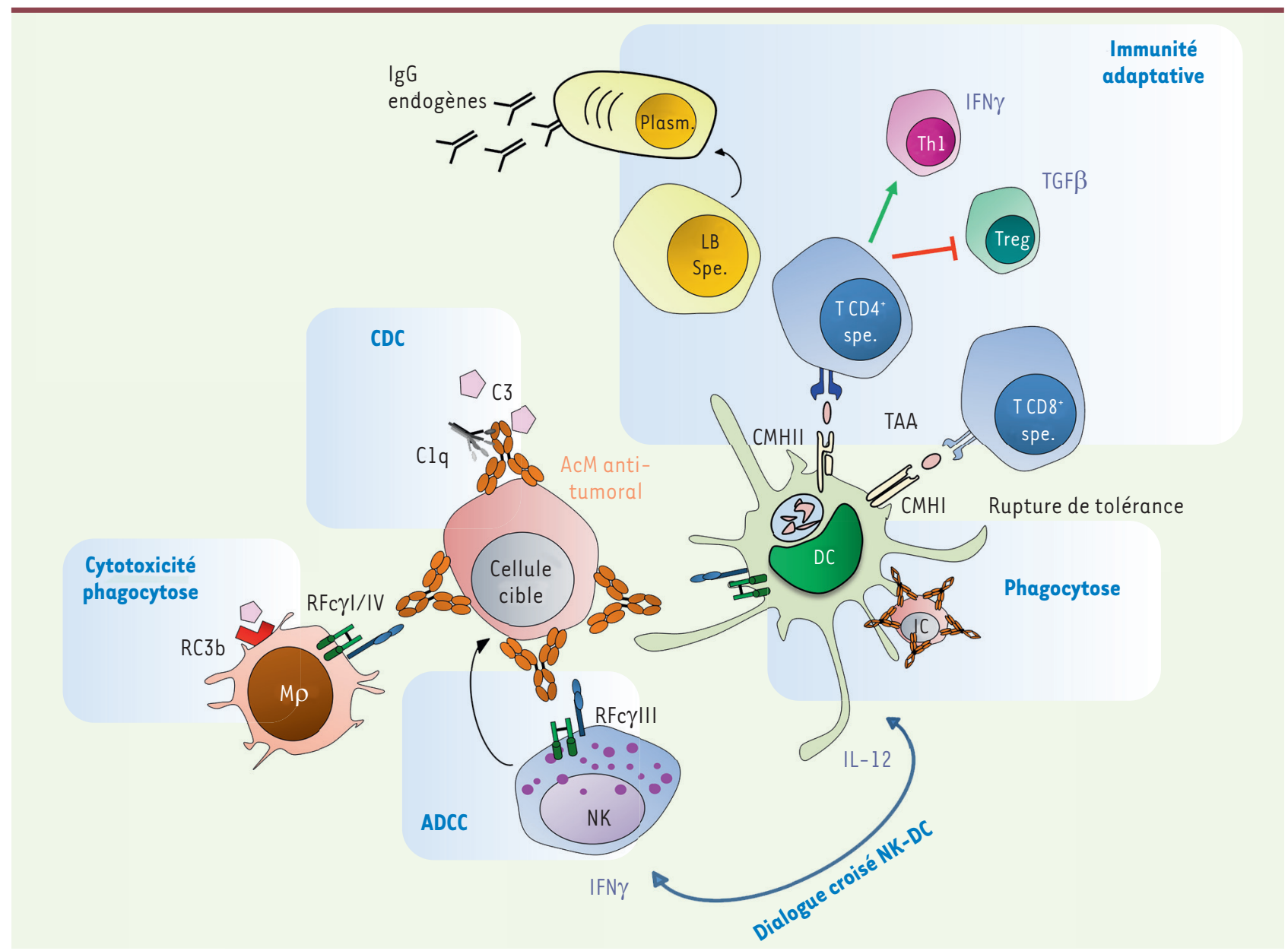

Figure 3. Mécanismes impliqués dans la mise en place d'une réponse immunitaire mémoire par les AcM anti-tumoraux. Les AcM anti-tumoraux ciblent spécifiquement les cellules tumorales et recrutent, par leur région Fc, les molécules du complément, les cellules NK ainsi que les macrophages qui induisent la lyse de la cellule tumorale respectivement par $C D C, A D C C$ et $A D C P$, ainsi que la formation de complexes immuns. Ces complexes immuns captés par les cellules présentatrices d'antigène exprimant les RFc $\gamma$ (cellules dendritiques, macrophages) sont à l'origine de l'initiation de la réponse innée. La production d'IFN(interféron)- $\gamma$ par les cellules NK (natural killer) et d'IL(interleukine)-12 par les cellules dendritiques et les macrophages dans le microenvironnement tumoral renforce leur activation respective par un mécanisme de dialogue croisé («crosstalk») positif. Ces premières étapes conduisent à une meilleure activation et maturation des cellules dendritiques, à une rupture de tolérance vis-à-vis d'antigènes du soi, et à une présentation antigénique optimale via les molécules du CMH (complexe majeur d'histocompatibilité) de classes I et II, permettant une activation de la réponse adaptative lymphocytaire T CD $4^{+}$et CD $8^{+}$spécifiques, avec une modification de la polarisation du système immunitaire d'un phénotype immunosuppressif T régulateur (Treg) vers un phénotype anti-tumoral Thl mémoire. La composante humorale de la réponse immunitaire se caractérise par la présence de lymphocytes B mémoires capables de produire des Ig spécifiques d'antigènes tumoraux formant des complexes immuns en absence d'anticorps thérapeutique. AcM : anticorps monoclonal. ADCC : cytotoxicité cellulaire dépendante des anticorps. ADCP : phagocytose dépendante des anticorps. $C D C$ : cytotoxicité dépendante du complément. $D C$ : cellule dendritique. IC : complexe immun. CMH : complexe majeur d'histocompatibilité. M $\rho$ : macrophage. RC3b : récepteur de la molécule du complément C3b. RFc $\gamma$ : récepteur de la région Fc des IgG. Spe. : spécifique. TAA : antigène associé aux tumeurs.

L'impact thérapeutique des AcM anti-tumoraux peut donc s'étendre à l'activation de lymphocytes $T$ spécifiques et à la mise en place d'un effet vaccinal. L'induction d'une immunité adaptative après une administration de courte durée d'un AcM a été observée dans le modèle de leucémie viro-induite cité précédemment, avec le développement d'une réponse mémoire humorale et cellulaire impliquée dans des effets protecteurs à long-terme [21]. La réponse humorale pourrait jouer un rôle essentiel dans la production d'immunoglobulines endogènes permettant de prendre le relais de l'anticorps thérapeutique, d'entretenir une boucle d'initiation de la réponse immunitaire par la formation de $\mathrm{Cl}$, et coopérer ainsi avec la réponse lymphocytaire T cytotoxique primordiale pour l'élimination des cellules 
cibles (Figure 3) [22, 23]. De tels mécanismes immunomodulateurs avaient également été montrés en 2010 dans un modèle murin de lymphome, dans lequel une déplétion des lymphocytes T CD4 ${ }^{+}$réduit dramatiquement l'effet anti-tumoral d'un anticorps anti-CD20 [24]. De même, la liaison de Cl impliquant cet AcM anti-CD20 au RFcylla exprimé par les cellules dendritiques conduit non seulement à la maturation de ces cellules mais aussi au développement d'une réponse immunitaire à long terme reposant sur les lymphocytes $T$ [25]. Des mécanismes immunomodulateurs similaires ont été observés dans des modèles précliniques de cancer du sein traités par un anticorps anti-Her2/neu, dans lequel la déplétion des lymphocytes T CD8 ${ }^{+}$abroge l'effet anti-tumoral de I'AcM [26]. La relation entre immunités innée et adaptative après traitement par des anticorps anti-tumoraux a été également démontré par la capacité d'un anticorps anti-Her2/neu à induire l'expression des molécules du complexe majeur d'histocompatibilité de classe II (CMHII) à la surface de cellules tumorales, en réponse à I'IFN- $\gamma$ produit par les macrophages activés par l'anticorps, conduisant à la reconnaissance directe des cellules tumorales par les lymphocytes $T$ CD $4^{+}[27]$.

De tels effets immunomodulateurs peuvent également participer au contrôle de la population lymphocytaire $C D 4^{+} T$ régulatrice immunosuppressive (Treg) [28]. Les Treg sont particulièrement représentés dans le microenvironnement de nombreuses tumeurs et il est probable que les AcM soient capables de moduler la polarisation et la spécificité de la réponse de ces lymphocytes. Dans un modèle murin, il a été notamment montré que l'activation des RFc $\gamma$ des cellules dendritiques par un AcM anti-CD20 est capable de réorienter, notamment via la production d'IL-12, la réponse immunosuppressive Trégulatrice vers un phénotype anti-tumoral de type Thl, producteur d'IFN- $\gamma$ [29]. Toujours dans le modèle murin de lymphome, il a été montré plus récemment qu'un traitement par un AcM anti-CD20 permettait le recrutement de macrophages et de cellules dendritiques mais aussi qu'il favorisait un processus de présentation antigénique croisée («cross-presentation ») par les cellules dendritiques, permettant d'induire des lymphocytes $T$ cytotoxiques spécifiques [30]. De tels résultats ont été obtenus chez des patients atteints de cancer et traités par des AcM anti-EGFR ou anti-HER2/neu, chez lesquels la fréquence des lymphocytes auto-réactifs reconnaissant des peptides dérivés de l'EGFR ou de HER2/neu augmente significativement après l'administration du traitement [4, 18], suggérant la capacité des AcM anti-tumoraux à favoriser une réponse immunitaire spécifique de l'antigène qu'ils ciblent.

Malgré l'évidence de l'établissement d'une réponse immunitaire adaptative, les effets thérapeutiques durables des AcM utilisés en monothérapie ne se limitent encore qu'à un faible pourcentage de patients (10 à $20 \%$ ). Comprendre les mécanismes de résistance ou d'échappement et développer des stratégies thérapeutiques combinant des AcM anti-tumoraux avec des thérapies adjuvantes permettant de renforcer leurs effets immunomodulateurs, afin d'obtenir des rémissions durables chez un nombre plus important de patients, est un des défis annoncés de ces futures années.

\section{Un intérêt majeur dans le développement de combinaisons thérapeutiques}

Cette nouvelle vision des AcM ciblant la tumeur, capables d'induire une réponse immunitaire durable, contribue à la conception de nouvelles approches thérapeutiques. Outre certaines isoformes de RFc $\gamma$ se rencontrant chez certains patients non-répondeurs ou répondeurs partiels, d'autres mécanismes de résistance sont impliqués avec, notamment, des facteurs intrinsèques à la cellule tumorale (diminution ou perte d'expression de la cible antigénique, perte d'immunogénicité, mutations modifiant les voies de signalisation), mais aussi des facteurs extrinsèques liés au microenvironnement tumoral, parmi lesquels les mécanismes d'échappement à l'immuno-surveillance. Ainsi, différentes stratégies de combinaisons thérapeutiques permettant de renforcer ou révéler les effets immunomodulateurs des AcM anti-tumoraux sont actuellement étudiées dans des modèles expérimentaux ou testées en essais cliniques pour de nombreux types de cancers.

\section{Induire la mort immunogénique}

Le traitement de lymphomes par I'AcM anti-CD20 rituximab peut induire la libération de signaux de danger par les cellules tumorales [31]. De même, les AcM anti-EGFR sont à l'origine d'une réponse cytotoxique spécifique grâce à l'induction d'une mort cellulaire immunogénique (ICD) de la cellule tumorale ciblée, un mécanisme par lequel la mort de la cellule permet d'alerter le système immunitaire via les récepteurs de l'immunité innée, comme les TLR (toll-like receptor) [32]. Ces mécanismes se caractérisent par le relargage, lors de la mort de la cellule tumorale, de ligands du TLR4, tels que la protéine HMGBl (highmobility group box-1), I'ATP et/ou la calréticuline. Les travaux de ces dernières années ont également révélé la capacité de certaines chimiothérapies et certains protocoles de radiothérapies à induire cette mort cellulaire immunogénique. Si les effets immunogéniques de ces thérapies conventionnelles et de certains AcM anti-tumoraux, utilisés en monothérapie, semblent insuffisants pour obtenir des taux de réponses élevés, leur association dans des protocoles de thérapies combinées est en plein essor. En effet, dans la mesure où les cellules, qui expriment les RFc $\gamma$ expriment en général également TLR4, ce double signal transmis à ces cellules qui sont des cellules présentatrices d'antigènes peut favoriser leur maturation et la présentation antigénique aux lymphocytes T. Ainsi la combinaison thérapeutique d'un AcM anti-Her2/neu et d'une chimiothérapie peut non seulement engendrer 
une immunité adaptative, mais peut également moduler la mise en place de l'immunité anti-tumorale mémoire [26]. De même, alors que, dans un modèle de cancer colorectal, le traitement par l'anticorps anti-EGFR, le cétuximab, ou par une chimiothérapie ont peu d'impact sur la réponse immunitaire anti-tumorale suite à la mort de la cellule tumorale, leur combinaison thérapeutique déclenche une mort cellulaire immunogénique se traduisant par une phagocytose plus active et un recrutement de lymphocytes $T$ spécifiques [33]. L'importance de l'association radiothérapie/anti-CD20 a également été montrée par une étude dans laquelle le traitement par radiothérapie de cellules de lymphomes augmente l'efficacité thérapeutique d'un AcM [34]. De telles combinaisons s'étendent désormais à de nombreux autres cancers. Des essais cliniques sont en cours, associant différentes chimiothérapies avec un AcM anti-Her2/neu, le trastuzumab et un AcM anti-EGFR, le pertuzumab, dans les cancers gastriques et œsophagiens (NCT02205047 ; NCT02120911), urothéliaux (NCT02006667), de l'ovaire (NCT02004093) ou du poumon non à petites cellules (NCT02226757). Un autre essai clinique associant le cétuximab (un autre anti-EGFR) à la radiothérapie vise à évaluer l'apparition de bio-marqueurs de réponses dans le sang, ainsi que des modifications histologiques de la tumeur dans une cohorte de patients atteints de carcinome épidermoïde de l'œsophage (NCT02123381).

\section{Stimuler le système immunitaire}

Parmi les mécanismes immunosuppresseurs, l'expression de points de contrôle immunitaire $(\mathrm{PCl})$ est particulièrement impliquée dans l'échappement et la résistance des cellules tumorales aux thérapies. Dans le mélanome, I'utilisation en clinique de l'ipilimumab et du nivolumab, deux AcM bloquant respectivement CTLA-4 (cytotoxic T lymphocyte-associated protein 4) et PD-1 (programmed cell death 1), a constitué une des avancées majeures en triplant l'espérance de vie d'un certain nombre de patients. Ces deux inhibiteurs de points de contrôle immunitaire, ainsi que de nouveaux AcM bloquants, voient leur utilisation en clinique s'étendre désormais à de nombreux types de cancers, ouvrant des perspectives intéressantes afin de lever les résistances aux immunothérapies par des AcM anti-tumoraux. Chez des patients atteints de lymphome folliculaire en récidive, l'efficacité du blocage de l'axe PD-1/PD-Ll par ces AcM a été démontrée en combinaison avec le rituximab (anti-CD20), avec un taux de réponse complète de $52 \%$ pour la combinaison thérapeutique, comparé à un taux de $11 \%$ obtenu avec la monothérapie utilisant le rituximab seul [35]. Le blocage de l'axe CTLA-4/CD28 par l'ipilimumab en combinaison avec le rituximab révèle également une augmentation de l'efficacité chez les patients atteints de lymphome. De nombreux essais cliniques associant des inhibiteurs de points de contrôle immunitaire à d'autres AcM anti-tumoraux comme l'obinutuzumab (NCT02220842), le trastuzumab (NCT02129556; NCT02649686), le cétuximab (NCT02713373 ; NCT03608046 ; NCT01860430) sont en cours dans des cancers hématopoïétiques, comme le lymphome, ou des cancers solides, comme le cancer du sein, le cancer colorectal et le cancer de la tête et du cou. Les résultats cliniques obtenus démontrent clairement l'intérêt de lever des freins immunitaires pour potentialiser les effets immunomodulateurs des AcM anti-tumoraux. Cependant les mécanismes cellulaires et moléculaires à l'origine de ces effets sont encore mal connus. Une étude récente réalisée dans un modèle de mélanome murin, révèle qu'un traitement par un anticorps antiPD-1, administré après l'échappement à une immunothérapie utilisant un anticorps ciblant les cellules tumorales par l'AcM anti-TYRPl, est capable de contrôler la croissance tumorale et permet une augmentation de la survie des animaux [20]. Ces effets sont liés à une réactivation par l'AcM anti-PD-1 des fonctions effectrices de l'infiltrat lymphocytaire de la tumeur qui avaient été initiées au préalable par l'AcM anti-TYRPl. D'autres approches de thérapies combinées visent à stimuler la réponse immunitaire par l'association d'agonistes de molécules de co-stimulation. L'utilisation d'un AcM ciblant CD137 (4-1BB), un récepteur de co-stimulation de la famille des récepteurs du TNF $\alpha$, potentialise les effets vaccinaux d'un anticorps antiEGFR, le cétuximab [36], et d'un anticorps anti-HER2/ neu, le trastuzumab, dans un modèle murin de cancer du sein [37]. Ces travaux démontrent l'effet potentialisateur de cette combinaison thérapeutique, I'AcM anti-EGFR fixé à la cellule tumorale favorisant le recrutement des cellules NK, et I'AcM agoniste entraînant une augmentation de leur fonction cytolytique en se liant à CD137 exprimé par ces cellules [38]. De même, l'engagement de la voie de co-activation lymphocytaire CD27/CD70 par un AcM agoniste anti-CD27 entraîne une potentialisation des effets d'un anticorps anti-CD20, dans le modèle de lymphome B murin BCLl ( $B$-cell leukemia/lymphoma 1) [39]. La stimulation de la voie CD27 induit une activation concomitante des cellules NK et des lymphocytes T CD $8^{+}$, ainsi qu'une libération de chimiokines et d'IFN- $\gamma$ responsables du recrutement et de l'activation de cellules myéloïdes. Dans ce même modèle de lymphome, des effets synergiques associés à un contrôle tumoral durable ont été observés en associant un AcM agoniste de la molécule de co-stimulation 0X40/CD134, membre de la famille des EGFR (AcM anti-CD134), à un AcM anti-CD20 [40]. Là encore, les effets observés sont le résultat d'une augmentation de l'activité cytolytique des cellules NK associée à une augmentation de la production d'IFN- $\gamma$. Une autre étude a montré l'intérêt de stimuler les étapes précoces de la réponse immunitaire anti-tumorale dans le cadre de combinaisons thérapeutiques avec des AcM antitumoraux. La molécule CD47, particulièrement surexprimée par les cellules douées d'activité phagocytaire, déclenche un signal inhibiteur bloquant la phagocytose lorsqu'elle se fixe à son ligand, SIRP $\alpha$ (signal regulatory 
protein $\alpha$ ). Le ciblage de la voie CD47/SIRP $\alpha$ par un AcM augmente ainsi l'activité phagocytaire des cellules et potentialise les effets immunomodulateurs de l'AcM anti-TYRPl dans un modèle de mélanome chez la souris [41].

\section{Cibler les voies de signalisation}

Les facteurs intrinsèques à la cellule tumorale et notamment les mutations qui altèrent les voies de signalisation participant aux processus de division cellulaire, de survie et de migration, sont également impliqués dans la résistance aux traitements. Plusieurs molécules ciblant ces voies, appelées petites molécules, sont entrées dans l'arsenal thérapeutique avec, notamment, dans le mélanome, les inhibiteurs de B-Raf (dabrafénib, vémurafénib, encorafénib, bénimétinib) ou de MEK (mitogen-activated protein kinase kinase) (tramétinib), dans le lymphome folliculaire, la LLC, le cancer bronchique non à petites cellules, les inhibiteurs de tyrosine kinases (ibrutinib, imatinib, sunitinib, afatinib), et, dans le cancer colorectal et gastro-intestinal ainsi que dans le cancer du foie, les inhibiteurs de multi-kinases à plus large spectre (régorafénib, sorafénib). D'autres molécules bloquent l'effet des protéines anti-apoptotiques, comme le vénétoclax qui se fixe sur $\mathrm{Bcl}-2$ (B-cell lymphoma 2). Malgré des effets thérapeutiques souvent rapides de ces molécules utilisées en monothérapie, les résistances sont sévères et fréquentes. Leur association dans des protocoles de thérapies combinées avec d'autres thérapies, en particulier avec des AcM ciblant la tumeur, s'est accrue ces dernières années. Le vénétoclax associé aux anticorps anti-CD20 rituximab ou obinutuzumab montre une augmentation du taux de réponse très significative et soutenue dans le temps dans la LLC, en particulier chez des patients réfractaires à l'ibrutinib, un inhibiteur de la tyrosine kinase de Bruton [42]. L'ublituximab (un AcM anti-CD20) fait l'objet d'un essai clinique en combinaison avec l'ibrutinib dans les LNH (NCT02006485). D'autres essais cliniques actuellement en cours associent le cétuximab avec l'afatinib (NCT02020577) et le régorafénib (NCT02095054; NCT01973868) dans des tumeurs solides métastatiques pour lesquelles aucune thérapie conventionnelle n'est préconisée. La mise en place de modèles précliniques associant les petites molécules aux AcM ciblant les cellules tumorales sera une étape importante dans la caractérisation des mécanismes cellulaires et moléculaires impliqués dans de telles combinaisons thérapeutiques.

\section{Conclusion}

Il est clair que des rémissions durables ne peuvent s'obtenir par les thérapies anti-cancéreuses que par l'administration concomitante ou séquentielle de plusieurs molécules, parmi lesquelles les AcM antitumoraux ont un rôle important à jouer. Les études de combinaisons associant des thérapies immuno-stimulatrices et/ou inductrices de mort de la cellule tumorale à des AcM anti-tumoraux démontrent le bénéfice clinique de telles associations. Les premiers modèles d'études précliniques soulignent également l'intérêt d'associer des molécules adjuvantes dont le rôle est de renforcer les effets immunomodulateurs des AcM anti-tumoraux et d'augmenter l'efficacité de la réponse anti-tumorale dans le temps. Le développement d'autres modèles précliniques devrait permettre de caractériser les mécanismes cellulaires et moléculaires impliqués dans ces effets protecteurs à long-terme. La compréhension de ces mécanismes sera une étape importante pour répondre aux questions qui se posent désormais pour chaque type de cancer: quelles molécules administrer, à quels moments et à quelles doses, afin que chaque combinaison thérapeutique aboutisse à une dynamique de potentialisation de la réponse anti-tumorale. $\diamond$

\section{SUMMARY}

Anti-tumor monoclonal antibodies: new insights to elicit a long-term immune response

Tumor-targeting monoclonal antibodies (mAbs) are now widely used for the treatment of cancer patients and their numbers are constantly increasing. Over the past ten years, numerous studies have demonstrated that the anti-tumor role of these antibodies far exceeds that of passive therapies as it was initially described, with the possibility of recruiting innate immune cells to promote activation of the early stages of immune response and to generate a long-term protective antitumor memory immune response. Understanding these mechanisms has recently led to the clinical development of a new generation of anti-tumor antibodies modified to increase their ability to interact with immune cells. Finally, the first preclinical and clinical studies have recently demonstrated the interest of developing therapeutic combinations combining anti-tumor mAbs with immune-, chemo- or radiotherapy, to reinforce their immunomodulatory potential and ensure effective and durable anti-tumor protection. $\diamond$

\section{LIENS D'INTÉRÊT}

Les auteurs déclarent n'avoir aucun lien d'intérêt concernant les données publiées dans cet article.

\section{RÉFÉRENCES}

1. Miller RA, Maloney DG, Warnke R, Levy R. Treatment of B-cell lymphoma with monoclonal anti-idiotype antibody. N EnglJ Med 1982 ; 306 : 517-22.

2. Marshall MJE, Stopforth RJ, Cragg MS. Therapeutic antibodies: what have we learnt from targeting CD20 and where are we going? Front Immunoly 2017 ; $8: 1245$.

3. Cartron G, Watier H, Golay J, Solal-Celigny P. From the bench to the bedside: ways to improve rituximab efficacy. Blood $2004 ; 104: 2635-42$.

4. Taylor C, Hershman D, Shah N Augmented HER-2 specific immunity during treatment with trastuzumab and chemotherapy. Clin Cancer Res 2007 ; 13 : 5133-43.

5. Cartron G, Dacheux L, Salles G, et al. Therapeutic activity of humanized anti-CD20 monoclonal antibody and polymorphism in IgG Fc receptor FcgammaRIlla gene. Blood $2002 ; 99: 754-8$.

6. Musolino A, Naldi N, Bortesi B Immunoglobulin $G$ fragment $C$ receptor polymorphisms and clinical efficacy of trastuzumab-based therapy in patients with HER-2/neu-positive metastatic breast cancer. J Clin Oncol $2008 ; 26: 1789-96$.

7. Takahashi M, Kuroki $Y$, Ohtsubo K, Taniguchi N. Core fucose and bisecting $\mathrm{GlcNAc}$, the direct modifiers of the $\mathrm{N}$-glycan core: their functions and target proteins. $2009 ; 344: 1387-90$. 


\section{RÉFÉRENCES}

8. Abes R, Dutertre CA, Agnelli L, Teillaud JL. Activating and inhibitory Fcgamma receptors in immunotherapy: being the actor or being the target. Rev Clin Immunol 2009 ; 5 : 735-47.

9. Ratner M. Small biotech steers HDAC inhibitor to clinic. Nat biotechnol $2014 ; 32: 853-4$.

10. Mastrangeli R, Palinsky W, Bierau H. Glycoengineered antibodies: towards the next-generation of immunotherapeutics. Glycobiology $2019 ; 29: 199-210$.

11. Mossner $\varepsilon$, Brunker $P$, Moser $S$ Increasing the efficacy of $C D 20$ antibody therapy through the engineering of a new type II anti-CD20 antibody with enhanced direct and immune effector cellmediated B-cell cytotoxicity. Blood $2010 ; 115: 4393-402$.

12. Yu X, Marshall MJE, Cragg MS, Crispin M. Improving antibody-based cancer therapeutics through glycan engineering. BioDrugs $2017 ; 31$ : 151-66.

13. Sharman JP, Farber CM, Mahadevan D, et al. Ublituximab (TG-1101), a novel glycoengineered anti-CD20 antibody, in combination with ibrutinib is safe and highly active in patients with relapsed and/or refractory chronic lymphocytic leukaemia: results of a phase 2 trial. 2017 ; $176: 412-20$

14. Luo C, Chen S, Xu N, et al. Glycoengineering of pertuzumab and its impact on the pharmacokinetic/pharmacodynamic properties. Sci Rep $2017 ; 7: 46347$.

15. Wierda WG, Kipps TJ, Mayer J, et al. Ofatumumab as single-agent CD20 immunotherapy in fludarabine-refractory chronic lymphocytic leukemia. $2010 ; 28: 1749-55$.

16. Michaud HA, Gomard T, Gros L, et al. A crucial role for infected-cell/antibody immune complexes in the enhancement of endogenous antiviral immunity by short passive immunotherapy. $2010 ; 6$ : el000948.

17. Overdijk MB, Verploegen S, Bogels M Antibody-mediated phagocytosis contributes to the antitumor activity of the therapeutic antibody daratumumab in lymphoma and multiple myeloma. mAbs $2015 ; 7: 311-21$.

18. Srivastava RM, Lee SC, Andrade Filho PA, et al. Cetuximab-activated natural killer and dendritic cells collaborate to trigger tumor antigen-specific T-cell immunity in head and neck cancer patients. Clin Cancer Res $2013 ; 19: 1858-72$

19. Borg C, Jalil A, Laderach D NK cell activation by dendritic cells (DCs) requires the formation of a synapse leading to IL-12 polarization in DCs. Blood 2004 ; 104 : 3267-75.

20. They L, Michaud HA, Becquart 0, et al. PD-1 blockade at the time of tumor escape potentiates the immune-mediated antitumor effects of a melanoma-targeting monoclonal antibody. Oncoimmunology $2017 ; 6$ : el353857.

21. Gros L, Dreja H, Fiser AL, et al. Induction of long-term protective antiviral endogenous immune response by short neutralizing monoclonal antibody treatment. J Virol $2005 ; 79: 6272-80$.

22. Gros L, Pelegrin M, Michaud HA, et al. Endogenous cytotoxic T-cell response contributes to the long-term antiretroviral protection induced by a short period of antibody-based immunotherapy of neonatally infected mice. J Virol $2008 ; 82: 1339-49$.

23. Nasser R, Pelegrin M, Michaud HA, et al. Long-lasting protective antiviral immunity induced by passive immunotherapies requires both neutralizing and effector functions of the administered monoclonal antibody. J Virol $2010 ; 84: 10169-81$

24. Abes R, Gelize $\varepsilon$, Fridman WH, Teillaud JL. Long-lasting antitumor protection by anti-CD20 antibody through cellular immune response. Blood $2010 ; 116$ : 926-34.

25. DiLillo DJ, Ravetch JV. Differential Fc-receptor engagement drives an anti-tumor vaccinal effect. $2015 ; 161: 1035-45$

26. Park S, Jiang Z, Mortenson ED The therapeutic effect of anti-HER2/neu antibody depends on both innate and adaptive immunity. Cancer Cell $2010 ; 18$ : 160-70.
27. Mortenson ED, Park S, Jiang Z, et al. Effective anti-neu-initiated antitumo responses require the complex role of CD4+ T cells. Clin Cancer Res 2013 ; $19: 1476-86$

28. Nasser R, Pelegrin M, Plays M Control of regulatory T cells is necessary for vaccine-like effects of antiviral immunotherapy by monoclonal antibodies. Blood 2013; 121 : 1102-11.

29. Deligne C, Metidji A, Fridman WH, Teillaud JL. Anti-CD20 therapy induces a memory Thl response through the IFN-gamma/IL-12 axis and prevents protumor regulatory T-cell expansion in mice. $2015 ; 29: 947-57$.

30. Ren Z, Guo J, Liao J, et al. CTLA-4 limits anti-CD20-mediated tumor regression. Clin Cancer Res 2017 ; 23 : 193-203.

31. Zhao T, Ren H, Wang X, et al. Rituximab-induced HMGBl release is associated with inhibition of STAT3 activity in human diffuse large B-cell lymphoma. Oncotarget $2015 ; 6: 27816-31$.

32. Garrido G, Rabasa A, Sanchez B, et al. Induction of immunogenic apoptosis by blockade of epidermal growth factor receptor activation with a specific antibody. J Immunol $2011 ; 187: 4954-66$

33. Pozzi C, Cuomo A, Spadoni l, et al. The EGFR-specific antibody cetuximab combined with chemotherapy triggers immunogenic cell death. Nat med $2016 ; 22: 624-31$

34. Singh V, Gupta D, Arora R Surface levels of CD20 determine anti-CD20 antibodies mediated cell death in vitro. PloS one 2014 ; 9 : ell1113.

35. Westin JR, Chu F, Zhang M, et al. Safety and activity of PDl blockade by pidilizumab in combination with rituximab in patients with relapsed follicular lymphoma: a single group, open-label, phase 2 trial. Lancet Oncol $2014 ; 15: 69-77$

36. Kohrt HE, Colevas AD, Houot $R$, et al. Targeting CD 137 enhances the efficacy of cetuximab. J Clin Invest 2014 ; 124 : 2668-82.

37. Kohrt HE, Houot R, Weiskopf $K$, et al. Stimulation of natural killer cells with a CD137-specific antibody enhances trastuzumab efficacy in xenotransplant models of breast cancer. J Clin Invest 2012 ; 122 : 1066-75.

38. Houot R, Kohrt H. CDI37 stimulation enhances the vaccinal effect of antitumor antibodies. Oncoimmunol $2014 ; 3$ : e941740.

39. Turaj AH, Hussain K, Cox KL, et al. Antibody tumor targeting is enhanced by CD27 agonists through myeloid recruitment. 2017 ; 32 : 777-91.e6.

40. Turaj AH, Cox KL, Penfold CA Augmentation of CDI34 (OX40)-dependent NK anti-tumour activity is dependent on antibody cross-linking. Sci Rep 2018 $8: 2278$

41. Sockolosky JT, Dougan M, Ingram JR Durable antitumor responses to CD47 lockade require adaptive immune stimulation. Proc Natl Acad Sci USA 2016 ; 113 : ع2646-54

42. Jones JA, Mato AR, Wierda WG, et al. Venetoclax for chronic lymphocytic leukaemia progressing after ibrutinib: an interim analysis of a multicentre, open-label, phase 2 trial. Lancet Oncol $2018 ; 19$ : 65-75.

\section{TIRÉS À PART}

L. Gros



Abonnez-vous sur 